Nervenarzt $2021 \cdot 92: 50-56$ https://doi.org/10.1007/s00115-020-00960-7 Online publiziert: 17. Juli 2020

(c) Der/die Autor(en) 2020

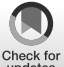

David Zilles-Wegner ${ }^{1}$ Sarah Trost ${ }^{1} \cdot$ Karoline Walliser $^{2} \cdot$ Leif Saager $^{2} \cdot$

\section{Sebastian Horn ${ }^{3}$. Mareike Ernst ${ }^{4}$}

${ }^{1}$ Klinik für Psychiatrie und Psychotherapie, Universitätsmedizin Göttingen, Göttingen, Deutschland

${ }^{2}$ Klinik für Anästhesiologie, Universitätsmedizin Göttingen, Göttingen, Deutschland

${ }^{3}$ Klinik für Kinderheilkunde und Jugendmedizin, Pädiatrische Kardiologie, Intensivmedizin und

Pneumologie, Universitätsmedizin Göttingen, Göttingen, Deutschland

${ }^{4}$ Klinik für Gynäkologie und Geburtshilfe, Universitätsmedizin Göttingen, Göttingen, Deutschland

\title{
Elektrokonvulsionstherapie in der Schwangerschaft: Fallbericht und interdisziplinäre Behandlungsvorschläge
}

\section{Hintergrund}

Schwerwiegende psychische Erkrankungen in der Schwangerschaft stellen einen relevanten Risikofaktor für Geburtskomplikationen dar. Nach einer aktuellen Registerstudie sind schizophrene und affektive Psychosen während der Schwangerschaft mit einer Vielzahl von mütterlichen, fetalen und neonatalen Komplikationen verbunden [28]. Neben immanent psychiatrischen Aspekten wie Suizidalität mit ggf. erweitertem Suizid sowie einer gestörten Mutter-Kind-Bindung umfassen diese ein erhöhtes Risiko für Sectio, ante- und postpartale Blutungen, vorzeitige Plazentalösung, Frühgeburten, Totgeburten und fetale Auffälligkeiten wie Wachstumsrestriktion und chronischer fetaler Stress.

Die Behandlung der psychischen Störung muss bei vorliegender Schwangerschaft in besonderem Maße Verträglichkeit und Sicherheit für Mutter und ungeborenes Kind gewährleisten. Zur Pharmakotherapie sind dazu Datenbanken wie etwa die des Pharmakovigilanzund Beratungszentrums für Embryonaltoxikologie (www.embryotox.de) verfügbar, die auf Grundlage von Fallberichten und -serien spezifische Empfehlungen zu einzelnen Wirkstoffen geben.

In manchen Fällen akuter psychiatrischer Störungen in der Schwangerschaft ist eine Psychopharmakotherapie nicht möglich, nicht gewünscht oder aber nicht ausreichend wirksam, sodass bei gegebener Indikation basierend auf entsprechenden Leitlinienempfehlungen [5-7] eine Elektrokonvulsionstherapie (EKT) in Betracht gezogen werden kann. Nationale wie internationale Leitlinien berücksichtigen die EKT in ihren Empfehlungen bei schwangeren Patientinnen und entsprechendem Schweregrad der Erkrankung. Die NICE-Guideline „Antenatal and postnatal mental health" etwa empfiehlt die EKT bei schwerer Depression, Manie, Mischzuständen oder Katatonie und zugleich bestehendem gesundheitlichem Risiko für Mutter oder Fetus [19]. Diese Empfehlungen basieren auf Fallsammlungen, die mangels der Möglichkeit randomisierter, kontrollierter Studien bei Schwangeren die bestmögliche Evidenz darstellen.

Die Indikationsstellung zur EKT in der Schwangerschaft ist trotz der Leitlinienempfehlungen mit Unsicherheiten verbunden und erfolgt daher nur selten. 2008 wurden für Deutschland lediglich fünf Behandlungen bei Schwangeren beschrieben [16]. Dies mag auch damit zusammenhängen, dass bisherige Übersichtsarbeiten zu sehr unterschiedlichen Schlussfolgerungen hinsichtlich der Sicherheit der EKT bei Schwangeren gelangen [23]. - Tab. 1 fasst die beschriebenen unerwünschten Ereignisse ohne Kausalitätsbeurteilung zusammen.
In einschlägigen Publikationen zur Therapie depressiver Störungen in der Schwangerschaft findet die EKT zum Teil keine Erwähnung [24]. In der deutschsprachigen Literatur fehlen konkrete Vorschläge zum praktischen Vorgehen [2], lediglich eine neuere internationale Publikation [26] beinhaltet Empfehlungen für das interdisziplinäre Management betroffener Patientinnen.

Ziel der aktuellen Arbeit ist es daher, basierend auf unserem konkreten Vorgehen im Einzelfall sowie den verfügbaren Literaturübersichten interdisziplinäre Vorschläge für die sichere Anwendung der EKT bei schwangeren Patientinnen $\mathrm{zu}$ erstellen.

\section{Methoden}

Neben der Darstellung des klinischen Falls werden Behandlungsvorschläge zum Vorgehen bei EKT in der Schwangerschaft aus den Fachdisziplinen Psychiatrie, Geburtshilfe, Anästhesiologie und Neonatologie gegeben. Diese haben nicht den Stellenwert von Leitlinienempfehlungen, sondern wurden im interdisziplinären Austausch aus der verfügbaren internationalen Literatur sowie den eigenen Erfahrungen im Sinne der guten klinischen Praxis abgeleitet. 
Tab. 1 In systematischen Reviews be-

schriebene unerwünschte Ereignisse unter

EKT in der Schwangerschaft ohne Kausali-

tätsbeurteilung. (Modifiziert nach [23])

\begin{tabular}{|c|c|}
\hline Mutter & $\begin{array}{l}\text { Fetus/ } \\
\text { Neugeborenes }\end{array}$ \\
\hline Prolongierte Anfälle & $\begin{array}{l}\text { Bradykardie/ } \\
\text { Arrhythmie }\end{array}$ \\
\hline $\begin{array}{l}\text { Uteruskontraktionen } \\
\text { ohne Frühgeburt }\end{array}$ & Frühgeburt \\
\hline $\begin{array}{l}\text { Abdominelle } \\
\text { Schmerzen }\end{array}$ & Abort/Totgeburt \\
\hline Vaginale Blutung & $\begin{array}{l}\text { Kongenitale Ano- } \\
\text { malien }\end{array}$ \\
\hline Plazentalösung & $\begin{array}{l}\text { Intrauterine Wachs- } \\
\text { tumsrestriktion }\end{array}$ \\
\hline Hämaturie & $\begin{array}{l}\text { Neonatales Atem- } \\
\text { notsyndrom }\end{array}$ \\
\hline Präeklampsie & Mentale \\
\hline Sectio & Retardierung \\
\hline
\end{tabular}

\section{Fallbericht}

\section{Psychiatrie}

Die 18-jährige schwangere Patientin wurde mit einem schizomanischen Syndrom aufgenommen. Psychopathologisch bestand eine Wahnsymptomatik mit hoher Wahndynamik einschließlich einer $\mathrm{Ne}$ gierung der Schwangerschaft, formalgedanklich Ideenflucht und Gedankenabreißen, zum Teil Personenverkennungen und Ich-Störung im Sinne von Gedankeneingebungen. Der Affekt war teilweise aggressiv-gereizt, das Verhalten distanzlos, zum Teil bizarr, psychomotorisch agitiert mit reduziertem Schlafbedürfnis sowie Konzentrations- und Gedächtnisstörungen.

Es wurde eine Medikation mit Quetiapin $700 \mathrm{mg} / \mathrm{d}$ sowie $30 \mathrm{mg} / \mathrm{d}$ Diazepam etabliert. Dennoch kam es zu aggressiven Erregungszuständen mit Gefährdung des ungeborenen Kindes, indem die Patientin sich auf den Bauch schlug, sich auf den Bauch warf oder diesen gegen Wände quetschte. Es erfolgte die Unterbringung nach Betreuungsrecht. Mehrfach waren Fixierungsmaßnahmen und intramuskuläre Medikation erforderlich.

Aufgrund der Notwendigkeit eines raschen Ansprechens sowie schwangerschaftsbedingt eingeschränkter Pharmakotherapie stellten wir die Indikation zur EKT. Patientin und gesetzlicher Betreu- er willigten in die Behandlung ein. Ab der vollendeten 28. Schwangerschaftswoche (SSW) erfolgten neun EKTBehandlungen (3/Woche, bitemporale Elektrodenplatzierung) in Anwesenheit von Gynäkologie/Geburtshilfe, Anästhesiologie und Neonatologie. Hierunter kam es zu einer raschen Besserung der Psychopathologie. Psychotische Symptome, psychomotorische Unruhe und aggressives Verhalten nahmen ab, Fixierungsmaßnahmen waren nicht mehr erforderlich. Ein einmalig prolongierter Anfall unter EKT wurde mittels Midazolam komplikationslos beendet. Parallel dazu kam es einmalig zu einer selbstlimitierenden fetalen Bradykardie (siehe unten). Die Patientin berichtete subjektiv über leichte Gedächtnisstörungen. Weitere unerwünschte Wirkungen traten nicht auf.

Der Versuch einer pharmakologischen Erhaltungstherapie schlug fehl, da unter Quetiapin $1000 \mathrm{mg} / \mathrm{d}$ keine ausreichende Konzentration im therapeutischen Drug Monitoring erreicht wurde, am ehesten aufgrund schwangerschaftsassoziierter CYP3A4-Induktion [20, 27]. Parallel zur Umstellung auf Risperidon $7 \mathrm{mg} / \mathrm{d}$ erfolgten vier weitere EKT-Behandlungen, die letzte mit 33 SSW. Das schizomanische Syndrom war vollständig remittiert, entsprechend einem Wert von 1 auf der Clinical Global Impression Scale (CGI-I). Postpartal konnte die erreichte Stabilität unter Risperidon erhalten werden.

\section{Anästhesie}

Das regelhafte anästhesiologische Vorgehen für eine EKT außerhalb der Schwangerschaft beinhaltet eine intravenöse Narkoseinduktion. Das Narkotikum sollte einen raschen Wirkeintritt und eine kurze Wirkdauer besitzen und den induzierten Anfall möglichst gering beeinflussen. Zur Oxygenierung erfolgt nach Bewusstseinsverlust eine BeutelMasken-Beatmung, seltener auch via Larynxmaske. Hierbei unterstützt eine moderate Hyperventilation eine angemessene Krampfaktivität. Eine endotracheale Intubation erfolgt in der Regel nicht, da der Eingriff an sich kein erhöhtes Aspirationsrisiko bedeutet. Vor
Anfallsinduktion erfolgt eine Muskelrelaxation mittels eines kurz wirksamen Muskelrelaxans, in der Regel Succinylcholin [9].

Ab der 12.SSW besteht aufgrund einer verzögerten Magenentleerung ein erhöhtes Aspirationsrisiko. Zudem werden bereits in den ersten SSW die Schleimhäute vermehrt durchblutet mit Blutungs- und Ödemneigung der Schleimhäute im Bereich der oberen Atemwege und höherer Inzidenz für einen schwierigen Atemweg und erschwerter endotrachealer Intubation [22]. Weiterhin besteht bei Schwangeren eine niedrigere funktionelle Residualkapazität infolge des erhöhten intraabdominellen Drucks. Bei der Patientin wurde daher eine Nüchternheitszeit von sechs Stunden vor EKT streng eingehalten. Zur Pufferung des Magensafts erhielt sie zudem $30 \mathrm{ml}$ 0,3-molares Natriumcitrat oral vor Narkoseeinleitung, zur Prophylaxe einer Hypersalivation Glycopyrroniumbromid i.v. (0,2 mg).

Die Einleitung der Allgemeinanästhesie erfolgte im Zentral-OP in Notsectiobereitschaft [10]. Es erfolgte eine leichte Linkslagerung und Optimierung des intravasalen Volumenstatus vor Beginn der EKT. Zielparameter war ein arterieller Mitteldruck von mindestens $65 \mathrm{~mm} \mathrm{Hg}$. In Rückenlage besteht ab der 16.-20. SSW das Risiko eines Vena-cavainferior-Kompressionssyndroms durch den graviden Uterus. Eine Kompression der Aorta abdominalis wiederum kann eine verminderte uterine Durchblutung mit möglicher fetaler Asphyxie bedingen. Die erwähnte Lagerung und Optimierung reduziert diese Risiken. Bei Bedarf wurde der Blutdruck durch Gabe von Akrinor ${ }^{\circledR}$ (ratiopharm $\mathrm{GmbH}$, Ulm, Deutschland) gesteigert.

Nach Präoxygenierung (exspiratorische Sauerstoffkonzentration $>75 \%$ ) erfolgte die Narkoseinduktion durch die i.v.-Gabe von Methohexital (initial $1 \mathrm{mg} / \mathrm{kg}$ Körpergewicht, im Verlauf benötigte die Patientin eine Dosissteigerung auf $1,6 \mathrm{mg} / \mathrm{kg}$ ). Mit Erreichen einer ausreichenden Narkosetiefe folgte die i.v.-Gabe von Succinylcholin $(1,5 \mathrm{mg} / \mathrm{kg}$ Körpergewicht). Neben der Schwangerschaft bestanden bei der Patientin keine Hinweise auf einen schwierigen Atemweg. Zugunsten einer kürzeren 
Narkosedauer und einer geringeren Invasivität bei absehbar regelmäßiger EKT mit vulnerablen Schleimhäuten entschieden wir uns trotz des Aspirationsrisikos gegen eine Atemwegssicherung mittels Endotrachealtubus. Nach Narkoseinduktion wurde eine druckkontrollierte Maskenbeatmung mit einem maximalen Beatmungsdruck von 15 mbar durchgeführt. Anders als bei nichtschwangeren Patienten wurde auf eine Normoventilation geachtet. Eine exzessive Hyperventilation kann durch Entstehung einer respiratorischen Alkalose und dadurch verminderte Sauerstoffabgabe vom maternalen an das fetale Blut zu einer fetalen Hypoxie führen [18, 26].

Bei einmalig prolongiertem Anfallsgeschehen wurde Midazolam i.v. (1,5 mg) verabreicht. Dies beeinträchtige das Aufwachverhalten der Patientin nicht. Ein erweitertes Atemwegsmanagement war nicht nötig.

\section{Gynäkologie und Geburtshilfe}

Einen wichtigen Aspekt in der Betreuung der Patientin stellte die regelmäßige Vorstellung in der Schwangerensprechstunde dar, durch die es gelang, ein Vertrauensverhältnis zwischen Patientin und behandelnden Gynäkologen/-innen aufzubauen. Dadurch konnten gemäß den aktuellen Mutterschaftsrichtlinien die vorgeschriebenen Vorsorgeuntersuchungen durchgeführt und im Mutterpass dokumentiert werden.

Aufgrund der Assoziation sowohl psychischer Erkrankungen als auch der EKT mit fetalen und maternalen Komplikationen (fetale Herzrhythmusstörungen, vorzeitiger Blasensprung, Uteruskontraktionen mit Eröffnung der Zervix, Plazentalösung und Frühgeburt $[4,26])$ erfolgte ein intensives CTGMonitoring je eine Stunde vor, kontinuierlich während sowie nach jeder EKT. Zudem wurden zum Ausschluss eines vorzeitigen Blasensprungs sowie einer Plazentalösung eine vaginale Inspektion mit Bestimmung des $\mathrm{pH}$-Werts beziehungsweise ein Amni-Check und eine Zervixlängenmessung nach jeder EKT durchgeführt. Diese Maßnahmen sind essentiell zum Erkennen einer drohenden Frühgeburt.

Nervenarzt 2021 · 92:50-56 https://doi.org/10.1007/s00115-020-00960-7

(c) Der/die Autor(en) 2020

D. Zilles-Wegner · S. Trost · K. Walliser $\cdot$ L. Saager · S. Horn · M. Ernst

\section{Elektrokonvulsionstherapie in der Schwangerschaft: Fallbericht und interdisziplinäre Behandlungsvorschläge}

\section{Zusammenfassung}

Hintergrund. Psychische Störungen in der Schwangerschaft sind häufig. Besonders bei schweren affektiven oder psychotischen Störungen mit Notwendigkeit eines raschen Ansprechens kann eine Elektrokonvulsionstherapie (EKT) indiziert sein. Dazu vorliegende Übersichtsartikel unterscheiden sich methodisch stark, was zu unterschiedlichen Schlussfolgerungen hinsichtlich der Anwendung der EKT bei Schwangerschaft führt.

Ziel der Arbeit. Darstellung eines neuen klinischen Falls sowie interdisziplinärer Behandlungsvorschläge zur sicheren Anwendung der EKT bei Schwangerschaft. Methoden. Fallbericht und selektive Literaturübersicht unter besonderer Berücksichtigung der existierenden systematischen Reviews. Ergebnisse und Diskussion. Die aktuelle Kasuistik zeigt die potenziell hohe Wirk- samkeit sowie die für Mutter und Fetus sichere Anwendung der EKT während der Schwangerschaft. Die in der Literatur beschriebenen unerwünschten Ereignisse entsprechen qualitativ weitgehend den Risiken bei schwerer psychischer Störung in der Schwangerschaft. Zur besseren NutzenRisiko-Abwägung wären größere Fall-Kontroll-Studien wünschenswert. Bei sorgfältiger Indikationsstellung, guter interdisziplinärer Abstimmung und Beachtung der Besonderheiten in der praktischen Durchführung ist die EKT auch in der Schwangerschaft eine sinnvolle Behandlungsoption.

\section{Schlüsselwörter}

Anästhesie · Geburtshilfe · Neonatologie · Wirksamkeit $\cdot$ Sicherheit

\section{Electroconvulsive therapy in pregnancy: case report and interdisciplinary treatment suggestions}

\section{Abstract}

Background. Psychiatric disorders during pregnancy are common. Electroconvulsive therapy (ECT) can be indicated in severely affective or psychotic disorders with the necessity of a rapid response. Currently available review articles greatly differ in the methodology, leading to divergent conclusions concerning the use of ECT during pregnancy.

Objective. Description of a new clinical case and interdisciplinary treatment suggestions for the safe application of ECT in pregnancy. Methods. Clinical case report and selective review of the literature with special consideration of existing systematic reviews. Results and conclusion. This case report shows the potentially high effectiveness and safe administration of ECT in pregnancy for both mother and fetus. The undesired adverse events associated with ECT described in the literature are largely qualitatively congruent with the risks of severe psychotic disorders in pregnancy per se. For a better riskbenefit analysis, larger case control studies would be desirable. Under the premise of a thorough evaluation of the indications, good interdisciplinary coordination and consideration of the specific practical requirements, ECT is a useful therapeutic option in pregnancy.

\section{Keywords}

Anesthesia - Obstetrics · Neonatology . Effectiveness · Safety
Zur Verhinderung eines Atemnotsyndroms im Fall einer Frühgeburt erfolgte vor der ersten EKT mit 28 SSW eine Lungenreifeinduktion $(2 \times 12 \mathrm{mg}$ Betamethason i.m. im Abstand von 24h; [3]). Bedingt durch das Risiko einer Frühgeburt sollten die Planung und Durchführung jeder EKT bei schwangeren Patientinnen interdisziplinär und unter Notsectiobereitschaft erfolgen [10].

Hinsichtlich der einmalig aufgetretenen fetalen Bradykardie handelte es sich gemäß der CTG-Bewertung nach FIGO um eine variable Dezeleration $<3$ min, die definitionsgemäß als suspekt einzustufen ist. Da sich im Anschluss wieder eine normale fetale Herzfrequenz mit 


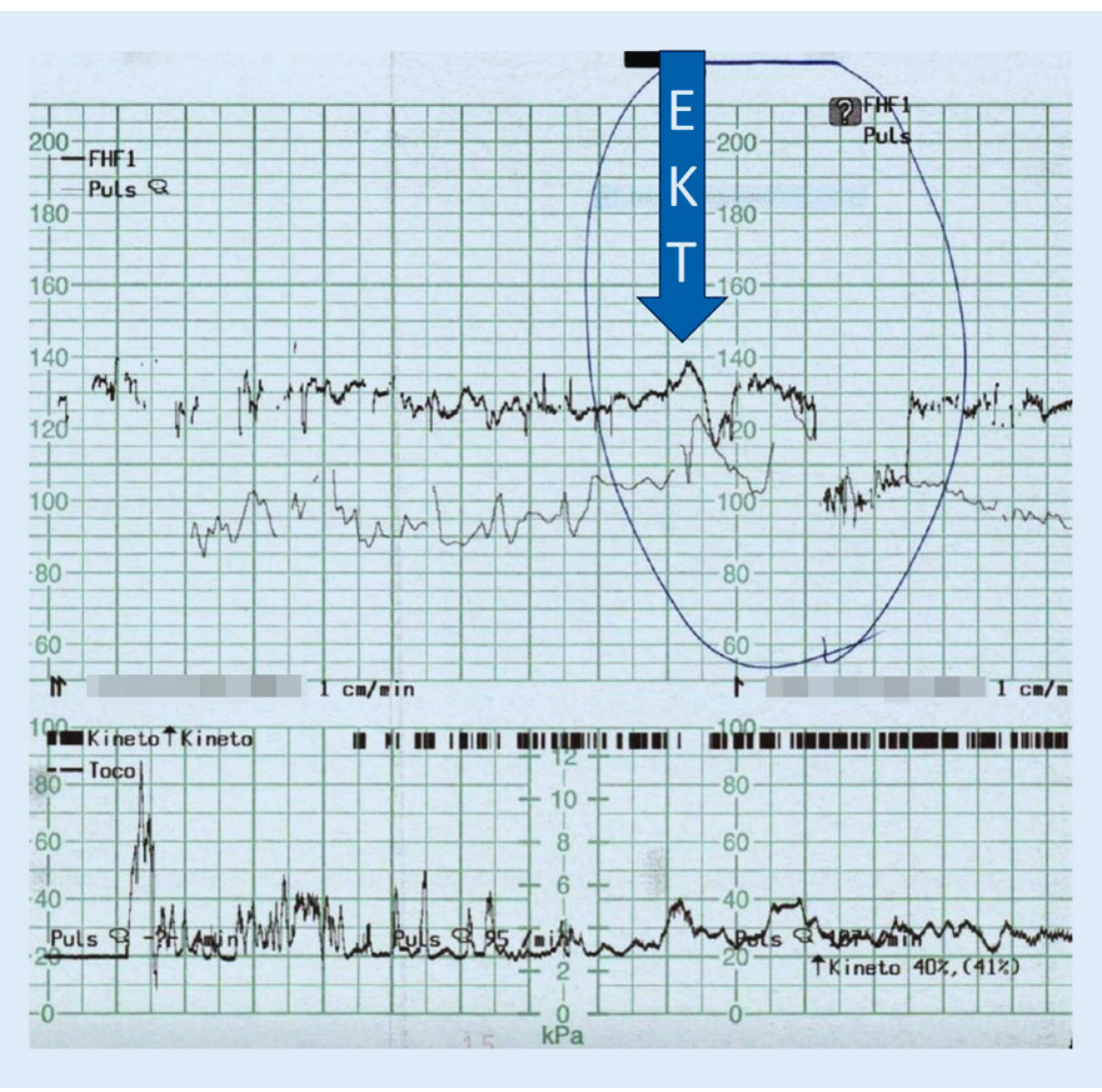

Abb. 1 ॥ CTG-Beschreibung: Baseline-Abfall während der EKT von 130 bpm auf 100 bpm über eine Dauer von 1,5 min. Anschließend Anstieg der fetalen Herzfrequenz auf 130 bpm mit eingeengtem Oszillationsmuster. Aufgrund der selbstlimitierenden Bradykardie von $<3$ min bestand kein Handlungsbedarf

regelrechtem Oszillationsmuster darstellte, bestand kein Handlungsbedarf (• Abb. 1).

Gemeinsam mit der Patientin entschieden wir uns aufgrund maternaler Erschöpfung mit ausgeprägter Angst vor einem Spontanpartus für eine primäre Sectio mit 37 SSW. Allgemein stellt die EKT keine Kontraindikation für eine Spontangeburt dar.

\section{Neonatologie}

Anders als in klassischen Situationen der neonatologischen Beratungs- und Betreuungssituation konnte in diesem Fall kein adäquates pränatales „counseling“ der Mutter durchgeführt werden. Die durch die Grunderkrankung der Mutter eingeschränkte Kommunikationsmöglichkeit bei unzureichender Compliance bot keine Grundlage, über die etwaigen Risiken einer schwangerschaftsbegleitenden EKT für den $\mathrm{Fe}$ -

\section{Ergebnisse}

\section{Klinischer Fall}

Im dargestellten Fall kam es unter EKT $\mathrm{zu}$ einer Remission des schizomanischen Syndroms inklusive des eigenund fremdgefährdenden Verhaltens. Ein prolongierter Anfall der Patientin unter EKT konnte unkompliziert pharmakologisch beendet werden, eine einmalige kurzfristige fetale Bradykardie sistierte spontan, ansonsten traten keine relevanten unerwünschten Wirkungen auf. Das Kind wurde mittels geplanter Sectio mit 37 SSW gesund geboren und zeigte nach neonatologischer Untersuchung während des kurzfristigen stationären Aufenthalts keine organischen Auffälligkeiten und eine reifeund altersentsprechende Entwicklung.

\section{Vorschläge zum interdisziplinären Management bei EKT in der Schwangerschaft}

Basierend auf den Erfahrungen der erfolgreichen Behandlung im oben dargestellten Fall sowie den zitierten systematischen Übersichtsarbeiten sollen nachfolgend tabellarisch Behandlungsvorschläge für die Durchführung der EKT in der Schwangerschaft formuliert werden (• Tab. 2). aufzuklären. Daher erfolgte pränatal prioritär die Bereitstellung einer dem aktuellen Reifegrad des Fetus angepassten Erstversorgungsumgebung in den jeweiligen Operationseinheiten. Durch den Einsatz einer vollständig mobilen Erstversorgungseinheit als Inkubatormikroumgebung (Giraffe OmnibedShuttle ${ }^{\circledR}$ Einheit, Fa. GE) konnte auf die variable Gesundheitssituation der Mutter und den jeweiligen Therapieort der EKT kurzfristig eingegangen werden.

Bereits während der pränatalen Therapiephase ist zudem eine interdisziplinäre Planung der zu erwartenden Betreuungssituation des Neugeborenen durchzuführen. Ein Bindungsaufbau zwischen Mutter und Kind muss koordiniert und kann stufenweise und unter Wahrung der Sicherheit des Kindes auch im Setting einer Neugeborenen-IMC-Station etabliert werden.

\section{Diskussion}

Unser Fallbericht bestätigt, dass die EKT auch in der Schwangerschaft eine gut wirksame sowie für Mutter und Fetus tion darstellen kann. Prinzipiell deckt sich diese Schlussfolgerung mit der verfügbaren Literatur. Sämtliche aktuellen deutschen Leitlinien für die Indikationen unipolare Depression, bipolare affektive Störung und Schizophrenie [5-7] benennen die EKT als therapeutische Option in der Schwangerschaft, jedoch mit der Einschränkung einer engen Indikationsstellung, sorgfältiger Nutzen-RisikoAbwägung sowie Vorsichtsmaßnahmen („in Zentren der Maximalversorgung mit entsprechender Erfahrung in der Durchführung der EKT“ [7]). Diese Empfehlungen basieren auf vier syssichere und verträgliche Therapieop- 
Psychiatrie Aufklärung über Diagnose, Behandlungsindikation, Therapieoptionen unter besonderer Berücksichtigung der Sicherheit für Mutter und Fetus. Abwägen der Chancen und Risiken der Therapie mit den nachteiligen Effekten der unbehandelten psychischen Störung

Die Auswahl der Behandlungsparameter (Elektrodenposition, Stimulationsdosis, Pulsbreite etc.) unterscheidet sich bei Schwangeren nicht von den allgemeinen Prinzipien [8]

Interdisziplinäre Durchführung mit Präsenz von Psychiatrie, Anästhesie, Gynäkologie/Geburtshilfe, Neonatologie

Gynäkologie/ Vor Beginn derEKT-Serienbehandlung

Geburtshilfe Adäquate präpartale Versorgung:

- Anlegen bzw. Vervollständigen des Mutterpasses

- regelmäßige Vorstellung in Schwangerensprechstunde

- frühzeitige Planung des Geburtsmodus zwischen Patientin, Geburtshilfe und Psychiatrie (Spontanpartus versus primäre Sectio; Spontanpartus bei guter Compliance möglich und primär angestrebt)

Qualifizierter Ultraschall mit Bestimmung von

- fetalem Gewicht

- Fruchtwassermenge

- plazentarer Versorgung (Dopplersonographie)

- Zervixlänge (Verkürzung und damit verbundenes Frühgeburtsrisiko)

CTG zur Ermittlung des fetalen Zustands (Herzfrequenz und Kindsbewegungen) sowie maternaler Kontraktionen

Lungenreifeinduktion zwischen der 24+0. SSW und 34+0. SSW ( $2 \times 12 \mathrm{mg}$ Betamethason im Abstand von $24 \mathrm{~h}$ ) zur Risikoreduktion eines "respiratory distress syndrome"

Während bzw. nach jeder EKT

Notsectiobereitschaft

CTG-Monitoring $1 \mathrm{~h}$ vor, kontinuierlich während und $1 \mathrm{~h}$ nach EKT

Vaginale Inspektion zum Ausschluss von Blutung bzw. Blasensprung (pH-Indikatorpapier bzw. Amni-Check)

Anästhesio- Sorgfältige Anamnese

logie Hinweise auf eine erschwerte Atemwegssicherung (Mallampati-Klasse, Mundöffnung, Halswirbelsäulenbeweglichkeit, thyreomentale Distanz, bekannter schwieriger Atemweg in vorhergehenden Narkosen, Narkoseausweis vorhanden?)

Vor Beginn der EKT

Nüchternheitszeiten strikt einhalten

Gabe von Natriumcitrat per os

Frühzeitiges Anlegen eines i.v.-Zugangs und Gabe von kristalloider Infusion

Gabe von Glycopyrroniumbromid i.v. vor Narkoseinduktion

Während EKT

Leichte Linkslagerung

Suffiziente Präoxygenierung (exspiratorische Sauerstoffkonzentration $>75 \%$ )

Normoventilation via Beutel-Masken-Beatmung ( $\mathrm{P}_{\text {insp }}<20 \mathrm{mbar}$ ), Muskelrelaxation

Engmaschige Blutdrucküberwachung, ggf. Gabe von Akrinor ${ }^{\circledR}$ (arterieller Mitteldruck $>65 \mathrm{~mm} \mathrm{Hg}$ )

Nach EKT

Nebenwirkungsmanagement:

- Bei postinterventionellem Kopfschmerz Paracetamol als Mittel der Wahl. Alternativ Ibuprofen bis zur 28. Schwangerschaftswoche

- Bei Übelkeit ist Meclozin Mittel der Wahl (über Auslandsapotheken erhältlich). Alternativ vorübergehend Dimenhydrinat (nicht im 3. Trimenon bei vorzeitiger Wehentätigkeit)

Neonatologie Sicherstellung der Verfügbarkeit einer Maximalversorgung in jeder SSW inkl. adäquater Personalausstattung

Nutzung einer mobilen Versorgungs- und Transporteinheit bei wechselnden Interventionsorten (integrierte Erstversorgungs- und Transporteinheit inkl. T-Stück, Respirator und Gasversorgung)

Kenntnis über die während der EKT genutzten Anästhetika im Falle einer Sectio caesarea (Apnoerisiko des Neugeborenen)

Benennung eines klinischen Teams (pflegerisch \& ärztlich), welches die Interventionen möglichst kontinuierlich koordiniert/begleitet

tematischen Reviews [1, 15, 18, 21], die sich in ihrem methodischen Vorgehen relevant unterscheiden. Selbst zwei neuere und nahezu zeitgleich publizierte Arbeiten $[15,21]$ kommen aufgrund unterschiedlicher Einschlusskriterien und verschiedener Ansätze zur Bewertung von Komplikationen zu unterschiedlichen Risikoeinschätzungen [23]. Leiknes et al. [15] ließen in ihre Risikobewertung alle sog. „adverse events“ einfließen (d.h. alle unerwünschten Ereignisse unabhän- gig von einer möglichen Kausalität) und kommen zu einer sehr restriktiven Empfehlung der EKT bei Schwangerschaft im Sinne einer Ultima Ratio. Hingegen nahmen Pompili et al. [21] den Versuch einer Kausalitätsbeurteilung vor und 
fanden für viele Ereignisse keinen $\mathrm{Zu}$ sammenhang mit der EKT. Entsprechend positiver fällt die Beurteilung der EKT aus als effektive therapeutische Option in der Schwangerschaft mit geringen Risiken.

Letztlich sind die absoluten und relativen Risiken der EKT in der Schwangerschaft nicht exakt bezifferbar, da kontrollierte Studien, die mittels identisch schwer erkrankter Vergleichspopulationen die Effekte der psychiatrischen Störung von denen der Behandlung differenzieren könnten, fehlen. Eine ohne Kausalitätsprüfung vorgenommene Angabe der Häufigkeit unerwünschter Ereignisse bei EKT in der Schwangerschaft wird diese jedoch überschätzen, da die EKT-assoziierten „adverse events“ [23] qualitativ weitgehend deckungsgleich mit den Risiken einer schwerwiegenden psychischen Erkrankung in der Schwangerschaft [14, 28] sind (- Tab. 1). Einigkeit sollte bestehen, dass durch die elektrische Stimulation keine teratogenen Effekte zu erwarten sind. Eine theoretische Arbeit zeigte zudem, dass die modellhaft berechneten Feldstärken im Bereich des fetalen Gehirns selbst bei maximalen Geräteeinstellungen unter den Grenzwerten für elektromagnetische Felder liegen [13].

Aus neonatologischer Sicht stellt am ehesten die Anästhesie ein potenzielles Risiko für den Fetus dar. Zur Anwendung im letzten Trimenon der Schwangerschaft liegt ein Warnhinweis der FDA bezüglich der Hirnentwicklung des Fetus und des Früh- und Neugeborenen vor [11], der jedoch zugleich äußert, dass einzelne bzw. kurze $(<3 \mathrm{~h})$ Anästhesien wahrscheinlich keinen negativen Effekt auf Verhalten und Lernen des Kindes haben. Die FDA ergänzte, dass schwangere Frauen bei gegebener Indikation und kurzer Anästhesiedauer $(<3 \mathrm{~h})$ einen notwendigen Eingriff nicht verzögern sollten [12]. Letztlich ist die klinische Evidenz unzureichend, um einen neurotoxischen Effekt prolongierter oder wiederholter Anwendung von Anästhetika in Schwangerschaft und Neugeborenenperiode beim Menschen ausschließen zu können [25]. Eine größere prospektive Multicenter-RCT ließ keine signifikant häufigeren Auffälligkeiten mit 2 bzw. 5 Jahren nach Narkoseinterventionen erkennen [17], wobei diese Studie keine Behandlung von Schwangeren und keine Mehrfachnarkosen beinhaltete und somit nur eingeschränkt übertragbar ist.

Aufgrund der heterogenen Datenbasis und Schlussfolgerungen der Übersichtsarbeiten zu EKT in der Schwangerschaft ist es weiterhin sinnvoll, entsprechende Fallberichte und Fallserien zu publizieren. Zur besseren kausalen Einordnung möglicher unerwünschter Ereignisse bei Mutter und Fetus bzw. Kind wären Fall-Kontroll-Studien wünschenswert, die aus methodischen Gründen jedoch schwer zu realisieren sind.

In Übereinstimmung mit den Leitlinien besteht unter den Autoren interdisziplinärer Konsens hinsichtlich einer engen Indikationsstellung zur EKT in der Schwangerschaft mit besonders sorgfältiger Betrachtung von Diagnose/ Indikation, individueller Nutzen-RisikoAbwägung und therapeutischen Alternativen. Ist dies gegeben und werden die o.g. interdisziplinären Behandlungsvorschläge beachtet, gibt es jedoch nach unserer übereinstimmenden Beurteilung keinen Grund, bei vorliegender Schwangerschaft auf die EKT zu verzichten. Kommt wie im vorliegenden Fall auch noch eine unmittelbare Gefährdung für Mutter und Fetus durch Erregungszustände und selbstverletzendes Verhalten hinzu, sollte das NutzenRisiko-Verhältnis deutlich zugunsten der EKT ausfallen.

\section{Fazit für die Praxis}

- Die EKT kann auch in der Schwangerschaft eine wirksame und sichere Therapieoption bei schweren psychischen Störungen sein.

- Eine Indikation zur EKT in der Schwangerschaft besteht bei schwerer affektiver oder psychotischer Symptomatik, daraus resultierender Gefährdung von Mutter und/oder Fetus sowie fehlender Wirksamkeit, Verträglichkeit oder Umsetzbarkeit anderer Therapien.

- Die kasuistisch beschriebenen unerwünschten Ereignisse entsprechen qualitativ den allgemeinen Risiken bei schwerer psychischer Störung in der Schwangerschaft und sind daher in ihrer Kausalität unklar.

- Ein abgestimmtes interdisziplinäres Management zwischen Psychiatrie, Gynäkologie, Anästhesiologie und Neonatologie ist grundlegend für die sichere Durchführung.

- Die Publikation von weiteren Fallberichten und -serien erscheint zur Verbesserung der Evidenzgrundlage sinnvoll.

\section{Korrespondenzadresse}

PD Dr. David Zilles-Wegner

Klinik für Psychiatrie und Psychotherapie,

Universitätsmedizin Göttingen

Von-Siebold-Str. 5, 37075 Göttingen,

Deutschland

david.zilles@med.uni-goettingen.de

Funding. Open Access funding provided by Projekt DEAL.

\section{Einhaltung ethischer Richtlinien}

Interessenkonflikt. D. Zilles-Wegner, S. Trost, K. Walliser, L. Saager, S. Horn und M. Ernst geben an, dass kein Interessenkonflikt besteht.

Für diesen Beitrag wurden von den Autoren keine Studien an Menschen oder Tieren durchgeführt. Für die aufgeführten Studien gelten die jeweils dort angegebenen ethischen Richtlinien.

Open Access. Dieser Artikel wird unter der Creative Commons Namensnennung 4.0 International Lizenz veröffentlicht, welche die Nutzung, Vervielfältigung, Bearbeitung, Verbreitung und Wiedergabe in jeglichem Medium und Format erlaubt, sofern Sie den/die ursprünglichen Autor(en) und die Quelle ordnungsgemäß nennen, einen Link zur Creative Commons Lizenz beifügen und angeben, ob Änderungen vorgenommen wurden.

Die in diesem Artikel enthaltenen Bilder und sonstiges Drittmaterial unterliegen ebenfalls der genannten Creative Commons Lizenz, sofern sich aus der Abbildungslegende nichts anderes ergibt. Sofern das betreffende Material nicht unter der genannten Creative Commons Lizenz steht und die betreffende Handlung nicht nach gesetzlichen Vorschriften erlaubt ist, ist für die oben aufgeführten Weiterverwendungen des Materials die Einwilligung des jeweiligen Rechteinhabers einzuholen.

Weitere Details zur Lizenz entnehmen Sie bitte der Lizenzinformation auf http://creativecommons.org/ licenses/by/4.0/deed.de. 


\section{Literatur}

1. Anderson EL, Reti IM (2009) ECT in pregnancy: a review of the literature from 1941 to 2007. Psychosom Med 71:235-242

2. Bader A, Frisch U, Wirz-Justice A, Riecher-Rössler A (2010) Depression during pregnancy and its treatment. Nervenarzt 81:267-276

3. Berger R, Abele H, Bahlmann F et al (2019) Prevention and therapy of preterm birth. Guideline of the DGGG, OEGGG and SGGG (S2k level, AWMF registry number $015 / 025$, february 2019)—part 2 with recommendations on the tertiary prevention of preterm birth and the management of preterm premature rupture of membranes. Geburtshilfe Frauenheilkd 79:813-833

4. Coshal S, Jones K, Coverdale J, Livingston R (2019) An overview of reviews on the safety of electroconvulsive therapy administered during pregnancy. J Psychiatr Pract 25:2-6

5. DGBS e.V., DGPPN e.V. (2019) S3-Leitlinie zur Diagnostik und Therapie Bipolarer Störungen. Langversion

6. DGPPN, BÄK, KBV, AWMF (Hrsg) (2015) S3Leitlinie/Nationale VersorgungsLeitlinie Unipolare Depression - Langfassung, 2. Aufl. Version 5 (für die Leitliniengruppe Unipolare Depression)

7. DGPPN e. V. (Hrsg) (2019) S3-Leitlinie Schizophrenie. Langfassung, 2019, Version 1.0, zuletzt geändert am 15. März 2019. https://www.awmf. org/leitlinien/detail/ll/038-009.html (für die Leitliniengruppe). 14. Febr. 2020

8. Grözinger M, Conca A, Nickl-Jockschat Tet al (Hrsg) (2013) Elektrokonvulsionstherapie kompakt. Für Zuweiser und Anwender. Springer, Heidelberg

9. Grundmann U, Schneider SO (2013) Narkose zur Elektrokrampftherapie. Anaesthesist 62:311-322

10. https://www.awmf.org/uploads/tx_szleitlinien/ 087-001I S1 Perinatologische Versorgung 2015-05.pdf.Zugegriffen: 14. Febr. 2020

11. https://www.fda.gov/drugs/drug-safety-andavailability/fda-drug-safety-communicationfda-review-results-new-warnings-about-usinggeneral-anesthetics-and. Zugegriffen: 14. Febr. 2020

12. https://www.fda.gov/drugs/drug-safety-andavailability/fda-drug-safety-communicationfda-approves-label-changes-use-generalanesthetic-and-sedation-drugs. Zugegriffen: 14. Febr. 2020

13. Kibret B, Premaratne M, Sullivan C et al (2018) Electroconvulsive therapy (ECT) during pregnancy: quantifying and assessing the electric field strength inside the foetal brain. Sci Rep 8:4128

14. Kurki T, Hiilesmaa V, Raitasalo R et al (2000) Depression and anxiety in early pregnancy and risk for preeclampsia. Obstet Gynecol 95:487-490

15. Leiknes KA, Cooke MJ, Jarosch-von Schweder L et al (2015) Electroconvulsive therapy during pregnancy: a systematic review of case studies. Arch Womens Ment Health 18:1-39

16. Loh N, Nickl-Jockschat T, Sheldrick AJ, Grözinger M (2013) Accessibility, standards and challenges of electroconvulsive therapy in Western industrialized countries: a German example. World J Biol Psychiatry 14:432-440

17. McCann ME, de Graaff JC, Dorris L et al (2019) Neurodevelopmental outcome at 5 years of age after general anaesthesia or awake-regional anaesthesia in infancy (GAS): an international, multicentre, randomised, controlled equivalence trial. Lancet 393:664-677
18. Miller LJ (1994) Use of electroconvulsive therapy during pregnancy. Hosp Community Psychiatry 45:444-450

19. NICE (2014) Antenatal and postnatal mental health: clinical management and service guidance. NICE clinical guideline 192. www.nice.org.uk/ guidance/cg192. 14. Febr. 2020

20. Pinheiro EA, Wisner KL, Clark CT (2018) Quetiapine dose adjustments in pregnant and postpartum women with bipolar disorder. J Clin Psychopharmacol 38:89-91

21. Pompili M, Dominici G, Giordano G et al (2014) Electroconvulsive treatment during pregnancy: a systematic review. Expert Rev Neurother 14:1377-1390

22. Rocke DA, Murray WB, Rout CC, Gouws E (1992) Relative risk analysis of factors associated with difficult intubation in obstetric anesthesia. Anesthesiology 77:67-73

23. Sinha P, Goyal P, Andrade C (2017) A meta-review of the safety of electroconvulsive therapy in pregnancy.JECT 33:81-88

24. VigodSN, Wilson CA, HowardLM(2016) Depression in pregnancy. BMJ 352:i1547. https://doi.org/10. 1136/bmj.i1547

25. Vlisides P, Xie Z (2012) Neurotoxicity of general anesthetics: an update. Curr Pharm Des 18:6232-6240

26. Ward HB, Fromson JA, Cooper JJ et al (2018) Recommendations for the use ofECT in pregnancy: literature review and proposed clinical protocol. Arch Womens Ment Health 21:715-722

27. Westin AA, Brekke M, Molden E et al (2018) Treatment with antipsychotics in pregnancy: changes in drug disposition. Clin Pharmacol Ther 103:477-484

28. Zhong QY, Gelaye B, Fricchione GL et al (2018) Adverse obstetric and neonatal outcomes complicated by psychosis among pregnant women in the United States. BMC Pregnancy Childbirth 18:120 https://doi.org/10.1186/s12884-018-1750-0

\section{Psychiatrie Update Livestream: Innovativ und flexibel}

19. und 20. Februar aus Berlin 26. und 27. Februar aus Mainz

Umfangreiches Update-Wissen in schwierigen Zeiten: Erstmals können sich interessierte Ärztinnen und Ärzte für das komplette Psychiatrie-Update-Seminar zum innovativen Livestream anmelden. Seien Sie LIVE dabei, bequem von zu Hause oder aus der Klinik! Die Interaktivität wird durch einen Livechat ermöglicht und die Zertifizierung ist beantragt, sodass Sie auch bei virtueller Teilnahme Ihre wichtigen CME-Punkte erhalten.

Das Update-Konzept: Die wichtigsten Neuerungen des vergangenen Jahres aus der Psychiatrie werden unter der wissenschaftlichen Leitung von Prof. Dr. Dieter F. Braus (Wiesbaden), Prof. Dr. Dr. Andreas Heinz (Berlin), Prof. Dr. Sabine Herpertz (Heidelberg) und Prof. Dr. Werner Strik (Bern) kritisch selektiert, analysiert und zusammengefasst. Die Relevanz für Ihren Klinik- und Praxisalltag steht dabei im Vordergrund und der ausführlichen Diskussion über den Livechat mit den Referierenden wird viel Raum gegeben.

In diesem Jahr werden neben den Kerngebieten der Psychiatrie die Hot Topics "Digitale Psychiatrie und Psychotherapie«, »Evolutionäre Perspektive der Psychiatrie« und »Naturheilkunde" vorgestellt.

Zum Gesamtpaket der Teilnahme gehören neben den umfangreichen Seminarunterlagen als eBook der Download aller Vortragspräsentationen und die Vorträge im Nachgang als Video-on-Demand auf der Videoplattform »streamed-up.com«.

Weitere Informationen zu Programm Referenten und zur Anmeldung: www.psychiatrie-update.com

Veranstalter: med update $\mathrm{GmbH}$ Hagenauer Straße 53 65203 Wiesbaden 